\title{
Inverse problem of breaking line identification by shape optimization
}

\author{
Daria Ghilli, Karl Kunisch and Victor A. Kovtunenko,
}

\begin{abstract}
An inverse breaking line identification problem formulated as an optimal control problem with a suitable PDE constraint is studied. The constraint is a boundary value problem describing the anti-plane equilibrium of an elastic body with a stress-free breaking line under the action of a traction force at the boundary. The behavior of the displacement is observed on a subset of the boundary and the optimal breaking line is identified by minimizing the $L^{2}$-distance between the displacement and the observation. Then, the optimal control problem is solved by shape optimization techniques via a Lagrangian approach. Several numerical experiments are carried out to show its performance in diverse situations.
\end{abstract}

Keywords. Optimization of shapes, inverse problems, free boundary problems, fracture.

2010 Mathematics Subject Classification. 35R35, 49Q10, 65N21, 74RXX.

\section{Introduction}

The inverse problem of identification of a free interface (breaking line) from boundary measurements is investigated as shape optimization problem for a class of admissible shapes of the breaking line. It has implications for testing of cracks for inhomogeneities, and discontinuities of media [3, 13, 21,23].

The problem is formulated as a least squares problem with a possible regularization term as:

$$
J(u, \Gamma)=\frac{1}{2} \int_{\Gamma_{o}}(u-z)^{2} d x+\beta \eta(u),
$$

where $\Gamma_{o}$ is the observation part of the boundary $\partial \Omega$ of a domain $\Omega \subset \mathbb{R}^{d},(d \in$ $\mathbb{N}$ ), and $z$ is the observed datum. The displacement $u$ in $\Omega$ itself satisfies a variational principle consisting of minimization of the following energy functional

$$
\mathcal{E}(u, \Gamma)=\frac{1}{2} \int_{\Omega \backslash \Gamma}|\nabla u|^{2} d x-\int_{\Gamma_{N}} g u d s+\phi\left(\llbracket u \rrbracket_{\Gamma}\right),
$$

The project has received funding from the European Research Council (ERC) under the European Union's Horizon 2020 research and innovation programme (advanced grant No. 668998 OCLOC). 
where $\Gamma$ is the breaking line in $\Omega$, and $g$ is the traction force at $\Gamma_{N} \subset \partial \Omega$. For fixed $\Gamma$, minimization of $\mathcal{E}(u, \Gamma)$ in (1.2) with respect to feasible $u$ describes the forward PDE problem, whose well-posedness is insured by conditions imposed on $\phi$. For instance, in [14] an example for non-uniqueness in a hemi-variational inequality is given, which is caused by (1.2) with non-smooth (piecewise-linear) $\phi$.

Note that the PDE constraint obtained by the variational formulation describes the anti-plane equilibrium under the action of the traction force $g$ of an elastic body with a breaking line. The penalty $\eta$ in (1.1) is a regularizing term with a parameter $\beta \geq 0$. The function $\phi$ in (1.2) denotes the fracture energy released with the opening of the breaking line, which is active on the jump of the displacement $u$ on $\Gamma$, denoted by $\llbracket u \rrbracket_{\Gamma}$.

Typically, the function $\phi$ is non-smooth and non-convex. From the physical point of view, it describes dissipative, non-ideal contact phenomena at the breaking line due to cohesion, non-linear friction, drag forces, etc. For the mathematical modelling, analysis, and numerical treatment of related problems within the theory of hemivariational and pseudo-monotone variational inequalities, see [1,14,26,29]. In the cohesive case, a buried crack can be determined by the set of points in $\Gamma$ where an opening $\llbracket u \rrbracket_{\Gamma} \neq 0$ occurs. This is the complement to the closed part of the breaking line where $\llbracket u \rrbracket_{\Gamma}=0$, see the corresponding fracture modelling and numerics in [20]. This formulation readily extends brittle fracture to frictionaltype non-linear phenomena and demonstrates the importance of the problem of identifying the breaking line.

Now the optimal breaking line identification problem can be formulated in the following way

$$
\inf _{\Gamma \in \mathcal{G}} J(u, \Gamma) \quad \text { such that } \varepsilon_{u}(u, \Gamma)=0,
$$

where $\mathcal{G}$ denotes the set of admissible breaking lines and $\mathcal{E}_{u}$ implies the variation with respect to $u$. The identification of $\Gamma$ in (1.3) represents a shape optimization, which is a specific case of an inverse problem, see the survey [17]. We refer to [30] for existence theorems in shape optimization. Uniqueness cannot be guaranteed as is typical for nonconvex optimization problems. In the present paper we chose $\beta=0$ and $\phi \equiv 0$.

To this regard we mention that, for fixed $\Gamma$, in [9] and [10] non-linear models for quasi-static evolution of cohesive fractures are studied and numerically solved by a finite dimensional approach. The non-linearity of the models investigated in [9] and [10] stems from the non-convexity and non-smoothness of the term $\phi$ used to model the cohesive fracture energy. A very interesting (and challenging) problem for further work would be to solve the shape optimal control problem (1.3) with a non convex and non smooth $\phi \neq 0$ in (1.2). 
In the context of shape optimization theory, variations of geometry are expressed with the help of the shape (Eulerian) derivative of the objective based on the Hadamard structure theorem (see [5]). Using a Lagrangian approach (see [15]) applied to variational problems, explicit analytic representation of the shape derivative for free boundary problems were derived in [8, 16, 25], and in [24] for our specific case. Finally, we mention [6] where free discontinuity problems were approximated by $\Gamma$-convergence and solved numerically by an iterative thresholding algorithm.

Among the earlier contributions addressing shape optimization of cracks, we refer to [28] where $2 \mathrm{D}$ curved cracks were identified using an optimization approach, and to $[2,7]$ for sensitivity formulas at the singular crack tip. Within the variational theory of non-linear crack problems subject to the non-penetration condition $\llbracket u \rrbracket_{\Gamma} \geq 0$ (see [18]), we cite $[19,22]$ for the shape differentiability. It is important to note that singular solutions, that are $H^{1}$-functions but lacking the $H^{2}$ regularity at the tips of crack, are inherent in cracked domains with non-Lipschitz boundaries. However, our study relies on piece-wise smooth $H^{2}$-solutions (see Proposition 4.1), because the breaking line is assumed meeting the domain boundary and splitting it into polyhedric subdomains.

Starting with preliminaries in Section 2, we formulate rigorously the optimal breaking line identification problem in Section 3, and state its regularity in Section 4. For the computation of the shape derivative of the objective functional $J$ (see Section 5, Theorem 5.1), we use a Lagrangian approach. We compute the adjoint $v$ of the state variable $u$ and we write the shape optimization problem (1.3) as a min max problem of a suitable Lagrangian depending on $u$ and $v$. Then, the derivative of the Lagrangian is computed by shape optimization and differential calculus techniques. The vector fields along which we compute the derivative are called velocities and denoted by $\theta$.

The result of Theorem 5.1 shows that the shape derivative of $J$ is represented by four terms: one term defined by jumps of the variables across the breaking line $\Gamma$, the second one given on the traction part, a third term on the observation part of the boundary, and a last term on the part of the boundary under Dirichlet boundary conditions (see formula (5.10)). A suitable choice of the velocity, taking into account the geometric configuration, provides a negative sign of the derivative, and thus a descent direction for the numerical optimization purposes.

In Section 6 we propose an algorithm to identify the breaking line. The main structure is described in Algorithm 1. It is based on a gradient step procedure where the breaking line is updated by adding the velocity, which is derived from the shape derivative of the objective functional. The reference boundary value problem and its adjoint are approximated by a finite element method. The key point for the computation of the velocity is the term defined by the jumps of the 
gradient of the variables across the breaking line $\Gamma$. Its treatment is described in Algorithm 2.

In Section 7 the performance of our identification algorithm is shown for three numerical experiments carried out in diverse situations. Different shapes of the true breaking line, admissible velocities and traction forces are tested. In the first experiment one tip (the left tip) of the trial breaking lines is moved during the iterations along the Dirichlet boundary to the true breaking line tip. The results show that our scheme is able to recover also the shape of the true breaking line quite well and in few iterations. The second experiment takes into account the velocity in the traction part, that is, the right breaking line tip, which is no more fixed, but rather moved during the iterations along the Neumann boundary under traction force. While for the first two test cases, the breaking line geometry is represented in a local basis, for the third one non-local ansatz functions are used.

\section{Notation}

Let $\mathcal{P}(D)$ be the set of subsets compactly contained in $D \subset \mathbb{R}^{d}$, where $D$ is assumed to be open and bounded. Define for $0 \leq \alpha \leq 1$ the space of vector fields

$$
C_{c}^{0, \alpha}\left(D, \mathbb{R}^{d}\right):=\left\{\theta \in C^{0, \alpha}\left(D, \mathbb{R}^{d}\right) \mid \theta \text { has compact support in } D\right\} .
$$

For a given domain $\Omega \subset D$ with at least a piece-wise $C^{1}$ boundary $\partial \Omega$ with no singular points, we introduce the set of boundary-preserving fields

$$
C_{c}^{0, \alpha}(\partial \Omega):=\left\{\theta \in C_{c}^{0, \alpha}\left(D, \mathbb{R}^{d}\right) \mid \theta \cdot n=0 \text { on } \partial \Omega\right\}
$$

where $n$ is the outward unit normal vector to $\Omega$.

Consider a vector field $\theta \in C_{c}^{0,1}\left(D, \mathbb{R}^{d}\right)$ and the associated flow $\phi_{t}^{\theta}: \bar{D} \rightarrow$ $\mathbb{R}^{d}, t \in\left[0, t_{0}\right]$ defined for each $x \in \bar{D}$ as $\phi_{t}^{\theta}(x):=y(t)$, where $y:\left[0, t_{0}\right] \rightarrow \mathbb{R}^{d}$ solves the first order non-linear ODE

$$
\frac{d}{d t} y(t)=\theta(y(t)) \quad \text { for } t \in\left(0, t_{0}\right), \quad y(0)=x .
$$

We will use the simpler notation $\phi_{t}^{\theta}=\phi_{t}$.

Since $\theta \in C_{c}^{0,1}\left(D, \mathbb{R}^{d}\right)$ we have by Nagumo's theorem [27] that, for fixed $t \in$ $\left[0, t_{0}\right]$, the flow $\phi_{t}$ is a homeomorphism from $D$ into itself and maps boundary onto boundary and interior onto interior.

\section{Optimal breaking line identification problem}

We consider domains which arise from splitting a hold-all-domain $\Omega$ by means of an interface representing the breaking line $\Gamma$ into subdomains $\Omega^{+}$and $\Omega^{-}$. More- 
over, here is assumed that the graph of the breaking line can be described by a function. In the following we refer to specific representatives $\Omega$ and $\Gamma$ in $2 \mathrm{D}$.

Let $\Omega=(0,2) \times(-0.5,0.5) \subset \mathbb{R}^{2}$. Assume

$$
\Omega=\Omega^{+} \cup \Omega^{-} \cup \Gamma, \quad \Gamma=\partial \Omega^{+} \cap \partial \Omega^{-},
$$

where the breaking line $\Gamma \in \mathcal{G}$ and

$$
\begin{aligned}
& \mathcal{G}=\left\{\left(x_{1}, x_{2}\right) \in \Omega\right. \text { such that } \\
& \left.\qquad x_{2}=\psi\left(x_{1}\right), \psi \in C^{1}([0,2]), \psi^{\prime}(0)=0, \psi^{\prime}(2)=0\right\} .
\end{aligned}
$$

Let the Dirichlet and Neumann boundaries be

$$
\Gamma_{D}:=\{0\} \times[-0.5, \psi(0)) \cup\{0\} \times(\psi(0), 0.5], \quad \Gamma_{N}\left(:=\partial \Omega \backslash \Gamma_{D}\right):=\Gamma_{N}^{0} \cup \Gamma_{N}^{+} \cup \Gamma_{N}^{-},
$$

where

$$
\Gamma_{N}^{0}=\{2\} \times[-0.5, \psi(2)) \cup\{2\} \times(\psi(2), 0.5]
$$

and

$$
\Gamma_{N}^{+}=(0,2) \times\{0.5\}, \quad \Gamma_{N}^{-}=(0,2) \times\{-0.5\} .
$$

We note that $\Gamma_{D}$ and $\Gamma_{N}^{0}$ consist of two disjoint segments separated by the end points (tips) $\{0\} \times\{\psi(0)\}$ and $\{2\} \times\{\psi(2)\}$ of the breaking line $\Gamma$. Since $\Gamma$ belongs to both $\partial \Omega^{+}, \partial \Omega^{-}$, we will use the notation $\Gamma^{ \pm}$denoting it as part of $\partial \Omega^{ \pm}$respectively.

Consider the forward boundary value problem in $\Omega \backslash \Gamma$ :

$$
\begin{cases}\Delta u=0 & \text { in } \Omega^{+} \cup \Omega^{-}, \\ u=0 & \text { on } \Gamma_{D}, \\ \nabla u \cdot n=g & \text { on } \Gamma_{N} \\ \nabla u \cdot n^{ \pm}=0 & \text { on } \Gamma^{ \pm}\end{cases}
$$

where $g \in L^{2}\left(\Gamma_{N}\right)$, and $n^{ \pm}$stands for the unit outer normals to $\Omega^{ \pm}$. Denote by $H_{\Gamma_{D}}(\Omega \backslash \Gamma)=\left\{u \in H^{1}\left(\Omega^{ \pm}\right): u=0\right.$ on $\left.\Gamma_{D}\right\}$. Clearly (3.2) admits a unique variational solution $u \in H_{\Gamma_{D}}(\Omega \backslash \Gamma)$.

System (3.2) describes the anti-plane equilibrium of an elastic body with a stress-free breaking line under the action of the force $g$. Note that (3.2) can be written as the following variational formulation

$$
\left\langle\mathcal{E}_{u}(u, \Gamma), v\right\rangle=\int_{\Omega \backslash \Gamma} \nabla u \cdot \nabla v d x-\int_{\Gamma_{N}} g v d s,
$$


where the energy functional $\mathcal{E}$ is defined as follows

$$
\mathcal{E}(u, \Gamma)=\frac{1}{2} \int_{\Omega \backslash \Gamma}|\nabla u|^{2} d x-\int_{\Gamma_{N}} g u d s .
$$

Let the observation boundary $\Gamma_{o}$ be a subset of $\Gamma_{N}$ and let $z \in H^{2}\left(\Omega^{ \pm}\right)$be a given function.

We consider the following cost functional

$$
J(u, \Gamma)=\frac{1}{2} \int_{\Gamma_{o}}(u-z)^{2} d x,
$$

where $u$ is the solution to problem (3.2) corresponding to $\Gamma$ and $\Omega$, as described above. The optimal breaking line identification problem is defined as follows

$$
\inf _{\Gamma \in \mathcal{G}} J(u, \Gamma) \quad \text { such that } \mathcal{E}_{u}(u, \Gamma)=0 .
$$

If the observation $z$ is feasible, i.e. solves the problem (6.2) similar to (3.2), then (3.4) is trivially solvable with $J(z, \Gamma)=0$. An example for a non-unique solution to (3.4) can be given by $u=x_{1}$ with $g=0$ on $\Gamma_{N}^{ \pm}, g=1$ on $\Gamma_{N}^{0}$, and the measurement $z=x_{1}$ on $\Gamma_{o}=\Gamma_{N}^{+} \cup \Gamma_{N}^{-}$, such that $J(u, \Gamma)=0$ for any $\Gamma=$ $[0,2] \times\{c\}$ with $c \in(-0.5,0.5)$.

\section{Regularity and adjoint state}

We have the following regularity of the solution $u$ of problem (3.2).

Proposition 4.1. Let $\Omega$ and $\Omega^{ \pm}$be as described above and $\Gamma \in \mathcal{G}$. Then the solution $u \in H^{1}\left(\Omega^{ \pm}\right)$of (3.2) satisfies

$$
u \in H^{2}\left(\Omega^{ \pm}\right) .
$$

Proof. The proof is based on the fact that $\partial \Omega^{ \pm}$has no singular points as defined in [12], where we used that $\Gamma \in \mathcal{G}$. For a complete proof we refer to [12], Chapter 2.

Our goal is to differentiate the functional $J(u, \Gamma)$ with respect to $\Gamma$.

For this purpose we use a Lagrangian method which is based on the following Lagrangian

$$
\mathcal{L}(u, v, \Gamma)=J(u, \Gamma)-\left\langle\mathcal{E}_{u}(u, \Gamma), v\right\rangle .
$$

Note that problem (3.4) is equivalent to

$$
\min _{u \in H_{\Gamma_{D}}(\Omega \backslash \Gamma)} \max _{v \in H_{\Gamma_{D}}(\Omega \backslash \Gamma)} \mathcal{L}(u, v, \Gamma) .
$$


The adjoint state is given by the following boundary value problem in $\Omega \backslash \Gamma$ :

$$
\begin{cases}\Delta v=0 & \text { in } \Omega^{+} \cup \Omega^{-}, \\ v=0 & \text { on } \Gamma_{D}, \\ \nabla v \cdot n=u-z & \text { on } \Gamma_{o}, \\ \nabla v \cdot n=0 & \text { on }\left\{\Gamma_{N} \backslash \Gamma_{o}\right\} \cup \Gamma^{ \pm},\end{cases}
$$

where we set the normal $n=-n^{+}=n^{-}$on the breaking line faces $\Gamma^{ \pm}$. Further we will use the notation for the jump along $\Gamma$ (see [18], Section 1.4):

$$
\llbracket v \rrbracket=\left.v\right|_{\Gamma^{+}}-\left.v\right|_{\Gamma^{-}} .
$$

Denoting by $u, v$ the solution to (3.2) and (4.2) respectively, the optimal value functional $\Gamma \rightarrow J(u, \Gamma)$ satisfies the following identity

$$
J(u, \Gamma)=\mathcal{L}(u, v, \Gamma) .
$$

\section{Shape derivative via Lagrangian approach}

Let $\theta \in C_{c}^{0,1}(\partial \Omega)$ and $x \mapsto \phi_{t}: H_{\Gamma_{D}}(\Omega \backslash \Gamma) \mapsto H_{\Gamma_{D}}\left(\Omega \backslash \Gamma_{t}\right)$ be the associated perturbation flow. Define $\Gamma_{t}=\phi_{t}(\Gamma)$ such that $\Gamma_{0}=\Gamma$.

The Eulerian derivative of $J$ at $\Gamma$ in the direction of $\theta$, when the limit exists, is defined as

$$
\left.\frac{d}{d t} J\left(u_{t}, \Gamma_{t}\right) \theta\right|_{t=0}:=\lim _{t \rightarrow 0} \frac{J\left(u_{t}, \Gamma_{t}\right)-J(u, \Gamma)}{t},
$$

where $u=\left.u_{t}\right|_{t=0}$ and $u_{t}$ satisfies the state constraint

$$
\mathcal{E}_{u}\left(u_{t}, \Gamma_{t}\right)=0 .
$$

By (4.3) we have

$$
\left.\frac{d}{d t} J\left(u_{t}, \Gamma_{t}\right) \theta\right|_{t=0}=\left.\frac{d}{d t} \mathcal{L}\left(u_{t}, v_{t}, \Gamma_{t}\right) \theta\right|_{t=0} .
$$

By a change of variable we can write

$$
\mathcal{L}\left(u_{t}, v_{t}, \Gamma_{t}\right)=\tilde{\mathcal{L}}\left(t, u_{t} \circ \phi_{t}, v_{t} \circ \phi_{t}, \Gamma\right) .
$$

Specifically we have

$$
\begin{aligned}
\tilde{\mathcal{L}}\left(t, u_{t} \circ \phi_{t}, v_{t} \circ \phi_{t}, \Gamma\right) & =-\int_{\Omega \backslash \Gamma} A(t) \nabla\left(u_{t} \circ \phi_{t}\right) \nabla\left(v_{t} \circ \phi_{t}\right) d x \\
& +\int_{\Gamma_{N}} g^{t}\left(v_{t} \circ \phi_{t}\right) \xi(t) d s+\frac{1}{2} \int_{\Gamma_{o}}\left(\left(u_{t} \circ \phi_{t}\right)-z^{t}\right)^{2} \xi(t) d s,
\end{aligned}
$$


where $g^{t}=g \circ \phi_{t}, z^{t}=z \circ \phi_{t}$, the Jacobian matrix $D \phi_{t}=\left\{\left(\phi_{t}\right)_{i, j}\right\}_{i, j=1}^{2}$, and

$$
\xi(t):=\operatorname{det}\left(D \phi_{t}\right)\left|D \phi_{t}^{-T} n\right|, \quad A(t):=\operatorname{det}\left(D \phi_{t}\right) D \phi_{t}^{-1} D \phi_{t}^{-T} .
$$

Then by [5], Chapter 10, Thm. 5.1, we have

$$
\left.\frac{d}{d t} J\left(u_{t}, \Gamma_{t}\right) \theta\right|_{t=0}=\left.\frac{d}{d t} \tilde{\mathcal{L}}\left(t, u_{t} \circ \phi_{t}, v_{t} \circ \phi_{t}, \Gamma\right) \theta\right|_{t=0}=\frac{\partial}{\partial t} \tilde{\mathcal{L}}(0, u, v, \Gamma) .
$$

We simplify the notation and we write

$$
\tilde{\mathcal{L}}(t, u, v, \Gamma):=I_{1}(t)+I_{2}(t)+I_{3}(t),
$$

where

$$
\begin{gathered}
I_{1}(t)=-\int_{\Omega \backslash \Gamma}(A(t) \nabla u) \cdot \nabla v d x, \quad I_{2}(t)=\int_{\Gamma_{N}} g^{t} v \xi(t) d s, \\
I_{3}(t)=\frac{1}{2} \int_{\Gamma_{o}}\left(u-z^{t}\right)^{2} \xi(t) d s .
\end{gathered}
$$

By (5.1) we calculate $A^{\prime}(0)=(\operatorname{div} \theta) I-D \theta^{T}-D \theta$ and the derivatives

$$
\left.\frac{d}{d t} I_{1}(t)\right|_{t=0}=-\int_{\Omega \backslash \Gamma}\left[\left((\operatorname{div} \theta) I-D \theta^{T}-D \theta\right) \nabla u\right] \cdot \nabla v d x
$$

with the identity matrix $I$, and

$$
\left.\frac{d}{d t} I_{2}(t)\right|_{t=0}=\int_{\Gamma_{N}}\left(\nabla g \cdot \theta v+g v \operatorname{div}_{\Gamma_{N}} \theta\right) d s
$$

where the tangential divergence

$$
\operatorname{div}_{\Gamma_{N}} \theta=\left.\operatorname{div} \theta\right|_{\Gamma_{N}}-(D \theta n) \cdot n,
$$

and finally

$$
\left.\frac{d}{d t} I_{3}(t)\right|_{t=0}=\int_{\Gamma_{o}}\left(-(u-z)(\nabla z \cdot \theta)+\frac{1}{2}(u-z)^{2} \operatorname{div}_{\Gamma_{o}} \theta\right) d s
$$

where

$$
\operatorname{div}_{\Gamma_{o}} \theta=\left.\operatorname{div} \theta\right|_{\Gamma_{o}}-(D \theta n) \cdot n
$$


Consider the first term in the right side of (5.3) component-wise and integrate by parts to obtain

$$
\begin{aligned}
-\int_{\Omega \backslash \Gamma}(\operatorname{div} \theta \nabla u) \cdot \nabla v d x & =-\int_{\Omega \backslash \Gamma} \theta_{k, k} u_{j} v_{j} d x=\int_{\Omega \backslash \Gamma} \theta_{k}\left(u_{j, k} v_{j}+u_{j} v_{j, k}\right) d x \\
& -\int_{\partial \Omega} \theta_{k} u_{j} v_{j} n_{k} d s+\int_{\Gamma} \theta_{k} \llbracket u_{j} v_{j} \rrbracket n_{k} d s
\end{aligned}
$$

Another integration by parts on the second and third term in (5.3) implies that

$$
\begin{aligned}
\int_{\Omega \backslash \Gamma}\left(\theta_{j, k}+\theta_{k, j}\right) u_{k} v_{j} d x & =-\int_{\Omega \backslash \Gamma}\left(\theta_{k}\left(u_{k, j} v_{j}+u_{k} v_{j, j}\right)+\theta_{j}\left(u_{k, k} v_{j}+u_{k} v_{j, k}\right)\right) d x \\
& +\int_{\partial \Omega}\left(\theta_{k} u_{k} v_{j} n_{j}+\theta_{j} u_{k} v_{j} n_{k}\right) d s \\
& -\int_{\Gamma}\left(\theta_{k} \llbracket u_{k} v_{j} \rrbracket n_{j}+\theta_{j} \llbracket u_{k} v_{j} \rrbracket n_{k}\right) d s
\end{aligned}
$$

Since by the first of (3.2) and (4.2) we have

$$
-\int_{\Omega \backslash \Gamma}\left(\theta_{k} u_{k} v_{j, j}+\theta_{j} u_{k, k} v_{j}\right) d x=0,
$$

summing up (5.6) and (5.7) we obtain

$$
\begin{aligned}
\left.\frac{d}{d t} I_{1}(t)\right|_{t=0}= & \int_{\partial \Omega}\left(\theta_{k}\left(u_{k} v_{j} n_{j}-u_{j} v_{j} n_{k}\right)+\theta_{j} u_{k} v_{j} n_{k}\right) d s \\
& -\int_{\Gamma}\left(\theta_{k}\left(\llbracket u_{k} v_{j} \rrbracket n_{j}-\llbracket u_{j} v_{j} \rrbracket n_{k}\right)+\theta_{j} \llbracket u_{k} v_{j} \rrbracket n_{k}\right) d s .
\end{aligned}
$$

Then we express in compact form

$$
\begin{aligned}
\left.\frac{d}{d t} I_{1}(t)\right|_{t=0} & =\int_{\partial \Omega}\left(\theta \cdot\left(\nabla u \frac{\partial v}{\partial n}+\nabla v \frac{\partial u}{\partial n}\right)-(\nabla u \cdot \nabla v)(\theta \cdot n)\right) d s \\
& -\int_{\Gamma} \llbracket \theta \cdot\left(\nabla u \frac{\partial v}{\partial n}+\nabla v \frac{\partial u}{\partial n}\right)-(\nabla u \cdot \nabla v)(\theta \cdot n) \rrbracket d s .
\end{aligned}
$$

By (3.2) and (4.2) and since $\theta \cdot n=0$ on $\partial \Omega$, we get

$$
\begin{array}{r}
\left.\frac{d}{d t} I_{1}(t)\right|_{t=0}=\int_{\Gamma} \llbracket \nabla u \cdot \nabla v \rrbracket(\theta \cdot n) d s+\int_{\Gamma_{N}}(\theta \cdot \nabla v) g d s+\int_{\Gamma_{o}}(\theta \cdot \nabla u)(u-z) d s \\
+\int_{\Gamma_{D}} \theta \cdot\left(\nabla u \frac{\partial v}{\partial n}+\nabla v \frac{\partial u}{\partial n}\right) \cdot
\end{array}
$$


Gathering (5.2), (5.4), (5.5), (5.8), we have

$$
\begin{aligned}
\left.\frac{d}{d t} J\left(u_{t}, \Gamma_{t}\right) \theta\right|_{t=0} & =\int_{\Gamma_{N}}\left(\theta \cdot \nabla(g v)+g v \operatorname{div}_{\Gamma_{N}} \theta\right) d s \\
& +\frac{1}{2} \int_{\Gamma_{o}}\left(\theta \cdot \nabla\left[(u-z)^{2}\right]+(u-z)^{2} \operatorname{div}_{\Gamma_{o}} \theta\right) d s \\
& +\int_{\Gamma} \llbracket \nabla u \cdot \nabla v \rrbracket(\theta \cdot n) d s+\int_{\Gamma_{D}} \theta \cdot\left(\nabla u \frac{\partial v}{\partial n}+\nabla v \frac{\partial u}{\partial n}\right) d s .
\end{aligned}
$$

We can now state our main result.

Theorem 5.1. Under the previous notations and assumptions, we have

$$
\begin{aligned}
\left.\frac{d}{d t} J\left(u_{t}, \Gamma_{t}\right) \theta\right|_{t=0}=\left.\llbracket g v \rrbracket \theta_{\mathrm{t}}\right|_{\bar{\Gamma} \cap \Gamma_{N}}+ & \left.\frac{1}{2}(u-z)^{2} \theta_{\mathrm{t}}\right|_{\partial \Gamma_{o}}+\int_{\Gamma} \llbracket \nabla u \cdot \nabla v \rrbracket(\theta \cdot n) d s \\
& +\int_{\Gamma_{D}} \theta \cdot\left(\nabla u \frac{\partial v}{\partial n}+\nabla v \frac{\partial u}{\partial n}\right) d s, \quad(5.10)
\end{aligned}
$$

where the tangential velocity $\theta_{\mathrm{t}}:=\theta-n(\theta \cdot n)$.

Proof. Consider the decomposition of the gradient vector into the normal and the tangential components as follows $\nabla=n \frac{\partial}{\partial n}+\nabla_{\mathrm{t}}$. Since $\theta \cdot n=0$, it holds $\theta \cdot \nabla=\theta_{\mathrm{t}} \cdot \nabla_{\mathrm{t}}$ on $\partial \Omega$. Therefore, integrating along the boundary by formula (2.125) in [31], due to $\theta=n(\theta \cdot n)+\theta_{\mathrm{t}}$, we get

$$
\begin{aligned}
& \int_{\Gamma_{N}}\left(\theta \cdot \nabla(g v)+(g v) \operatorname{div}_{\Gamma_{N}} \theta\right) d s=\left.\llbracket g v \rrbracket \theta_{\mathrm{t}}\right|_{\bar{\Gamma} \cap \Gamma_{N}}, \\
& \quad \frac{1}{2} \int_{\Gamma_{o}}\left(\theta \cdot \nabla\left[(u-z)^{2}\right]+(u-z)^{2} \operatorname{div}_{\Gamma_{o}} \theta\right) d s=\left.\frac{1}{2}(u-z)^{2} \theta_{\mathrm{t}}\right|_{\partial \Gamma_{o}} .
\end{aligned}
$$

Then the claim follows by combining (5.9) and (5.11).

Based on Theorem 5.1, to get a descent direction for $J$, it suffices to set

$$
\begin{aligned}
\theta_{\mathrm{t}}=-\llbracket g v \rrbracket \text { on } \bar{\Gamma} \cap \Gamma_{N}, \quad \theta_{\mathrm{t}}=-\frac{1}{2}(u-z)^{2} \text { on } \partial \Gamma_{o}, \\
\quad \theta \cdot n=-\llbracket \nabla u \cdot \nabla v \rrbracket \text { on } \Gamma, \quad \theta=-\left(\nabla u \frac{\partial v}{\partial n}+\nabla v \frac{\partial u}{\partial n}\right) \text { on } \Gamma_{D},
\end{aligned}
$$


such that according to (5.10) we have

$$
\begin{aligned}
\left.\frac{d}{d t} J\left(u_{t}, \Gamma_{t}\right) \theta\right|_{t=0}=-\left.\llbracket g v \rrbracket^{2}\right|_{\Gamma \cap \Gamma_{N}}-\left.\frac{1}{4}(u-z)^{4}\right|_{\partial \Gamma_{o}} & -\int_{\Gamma} \llbracket \nabla u \cdot \nabla v \rrbracket^{2} d s \\
& -\int_{\Gamma_{D}}\left|\nabla u \frac{\partial v}{\partial n}+\nabla v \frac{\partial u}{\partial n}\right|^{2} d s<0 .
\end{aligned}
$$

This justifies to use $\theta$ as a descent direction. In formula (5.12) the orthogonality of $\Gamma$ to the boundary of $\Omega$ with $\frac{\pi}{2}$-angle is used according to the specific configuration (3.1), otherwise we have to prescribe $\theta \cdot n=0$ on the intersection of the closure of $\Gamma$ with the boundary of $\Omega$. It worth noting that our analysis states the descent property of the following iterative scheme (6.1) in any spatial dimension. Its convergence as a gradient algorithm is validated by numerical tests in $2 \mathrm{D}$, while $3 \mathrm{D}$ implementation is the subject of future work.

\section{Identification Algorithm}

The algorithm is based on a gradient step procedure, carried out in a main cycle where the current breaking line $\Gamma^{i}$ is updated by adding the velocity field $\theta\left(\Gamma^{i}\right)$, so that

$$
\Gamma^{i+1}=\Gamma^{i}+\tau \theta\left(\Gamma^{i}\right)
$$

This is described in Algorithm 1.

Algorithm 1. (Identification of breaking line)

1: Inputs: $M, M z, n g, n g z, K_{\max }$.

2: Generate a not uniform mesh $\mathcal{M}_{T}$ for the true fractured domain $\Omega_{T}$, giving the observation datum (see (6.2)) by distmesh $2 d$.

3: Initialize $\Gamma=\Gamma^{0}$ and $\Omega^{0}=\Omega \backslash \Gamma^{0}$

4: while Stopping criterion is not fulfilled do

5: generate mesh $\mathcal{M}^{i}$ for the current broken domain $\Omega^{i}=\Omega \backslash \Gamma^{i}$ by distmesh $2 d$.

6: $\quad$ Compute $u^{i}$ solving state equation (3.2) with $\Gamma=\Gamma^{i}$ and $\Omega^{i}=\Omega \backslash \Gamma^{i}$.

7: $\quad$ Interpolate $\mathcal{M}_{T}$ with $\mathcal{M}^{i}$ on $\Gamma_{o}$.

8: $\quad$ Compute $v^{i}$ solving (4.2) with $\Gamma=\Gamma^{i}$ and $\Omega^{i}=\Omega \backslash \Gamma^{i}$.

9: $\quad$ Compute $\theta\left(\Gamma^{i}\right)$ by Algorithm 2 and solving (5.10) with $\Gamma=\Gamma^{i}, u=u^{i}$ and $v=v^{i}$.

10: $\quad$ for $\mathrm{k}=1$ do Rescale $\theta\left(\Gamma^{i}\right)$ by $0.5 / \max \left(\left|\theta\left(\Gamma^{1}\right)\right|\right)$.

11: end for

12: $\quad$ Let $\Gamma^{i+1}=\Gamma^{i}+\tau \theta\left(\Gamma^{i}\right)$. 


\section{3: end while}

end

We generate a non-uniform mesh with discretization number $n g$ and the discretization $M$ of the breaking line $\Gamma$ with the MATLAB 2D Mesh generator using distance functions distmesh $2 d(\mathrm{ng}, \mathrm{M})$, and distmesh $2 d(\mathrm{ngz}, \mathrm{Mz})$ for meshing of the true fractured domain. The corresponding mesh-sizes are $h=1 / n g$ and $h z=1 / n g z$.

In the numerical experiments, $n g=20, n g z=40$, the number $m+1 \geq 2$ of the points $x^{0}, x^{1}, \ldots, x^{m}$ is set for $M$ and $M z$ in Step 1, the time step $\tau>0$ in Step 12 will be discussed later on. The starting breaking line $\Gamma^{0}$ is the segment $\left\{\left(x_{1}, 0\right): x_{1} \in[0,2]\right\}$. To solve the problem (6.2), (3.2), (4.2) we use linear finite elements. We follow precisely the implementation described in [4], section 3, paragraph 3.1.1, 3.1.2. The Dirichlet boundary condition is posed by assigning the function values at Dirichlet boundary nodes. The solutions of the boundary value problems are computed independently in the upper domain $\Omega^{+}$and in the lower domain $\Omega^{-}$.

The observation datum $z$ coincides with the value on the observation part $\Gamma_{o}$ for the solution of the problem in $\Omega \backslash \Gamma_{T}$ :

$$
\begin{cases}\Delta z=0 & \text { in } \Omega_{T}^{+} \cup \Omega_{T}^{-}, \\ z=0 & \text { on } \Gamma_{D}, \\ \nabla z \cdot n=g & \text { on } \Gamma_{N} \\ \nabla z \cdot n=0 & \text { on } \Gamma_{T}^{ \pm}\end{cases}
$$

where $\Gamma_{T}$ is the true breaking line, and $g$ is as above.

The velocity is computed and updated by Algorithm 1 for a fixed number of iterations $K_{\max }$. It is determined through formula (5.12), which depends on the gradients of the state $u$ and adjoint $v$. We next describe their computation. The precise construction of the velocity $\theta\left(\Gamma^{i}\right)$ in Step 12 will be explained later on for every numerical test. Let $E$ be the element of the piece-wise linear triangulation with vertices given by $x^{1}=\left(x_{1}^{1}, x_{2}^{1}\right), x^{2}=\left(x_{1}^{2}, x_{2}^{2}\right), x^{3}=\left(x_{1}^{3}, x_{2}^{3}\right)$, and

$$
u(x)=\sum_{i=1}^{3} u_{i} \phi_{i}(x),
$$

where $\left\{\phi_{i}\right\}_{i=1}^{3}$ are the basis function of the triangulation. Then the gradient

$$
\nabla u(x)=\left(\begin{array}{l}
b \\
c
\end{array}\right), \quad x \in E,
$$


where $u\left(x^{i}\right)=: u_{i}$ and

$$
b=\frac{\operatorname{det}\left(\begin{array}{ccc}
1 & x_{1}^{2} & x_{2}^{1} \\
u_{1} & u_{2} & u_{3} \\
1 & x_{1}^{3} & x_{2}^{3}
\end{array}\right)}{\operatorname{det}\left(\begin{array}{ccc}
1 & x_{2}^{1} & x_{2}^{1} \\
1 & x_{1}^{2} & x_{1}^{2} \\
1 & x_{1}^{3} & x_{2}^{3}
\end{array}\right)}, \quad c=\frac{\operatorname{det}\left(\begin{array}{ccc}
1 & x_{1}^{1} & x_{2}^{1} \\
1 & x_{1}^{2} & x_{2}^{2} \\
u_{1} & u_{2} & u_{3}
\end{array}\right)}{\operatorname{det}\left(\begin{array}{ccc}
1 & x_{1}^{1} & x_{2}^{1} \\
1 & x_{1}^{2} & x_{1}^{2} \\
1 & x_{1}^{3} & x_{2}^{3}
\end{array}\right)} .
$$

For its proof we refer to [11], paragraph 4. In particular formula (6.3) is used in Algorithm 2.

\section{Algorithm 2. (Gradient of breaking line)}

1: Inputs: $u, v, \mathcal{M}, M, z$, where $\mathcal{M}=\left(\mathcal{M}^{+}, \mathcal{M}^{-}\right)$is the current mesh in $\Omega^{+}, \Omega^{-}$.

2: for $y \in M$ do

3: $\quad$ Find the correspondent points $\left(x_{y}^{+}, x_{y}^{-}\right) \in\left(\mathcal{M}^{+}, \mathcal{M}^{-}\right)$that are nearest to $y$ by the $\ell^{1}$ distance. Let $X_{y}^{-}=\left\{x_{y}^{-}\right\}_{y \in M}, X_{y}^{+}=\left\{x_{y}^{+}\right\}_{y \in M}$.

\section{4: end for}

5: for $x_{y}^{-} \in X_{y}^{-}$do

6: $\quad$ On the mesh $\mathcal{M}^{-}$, find the points $z_{y, l}^{-}, z_{y, r}^{-} \in \mathcal{M}^{-} \cap \Gamma$, that are nearest to $x_{y}^{-}$, denoting respectively the one on the left and the one of the right to $x_{y}^{-}$.

7: $\quad$ Find the elements $E_{y, l}^{-}, E_{y, r}^{-}$such that $x_{y}^{-}, z_{y, l}^{-} \in E_{y, l}^{-}$and $x_{y}^{-}, z_{y, r}^{-} \in$ $E_{y, r}^{-}$.

8: end for

9: for $x_{y}^{+} \in X_{y}^{+}$do

10: $\quad$ Repeat the same as in the previous cycle on the mesh $\mathcal{M}^{+}$.

11: end for

12: for $y \in M$ do

13: $\quad$ Compute $\nabla u(y), \nabla v(y)$ in $E_{y, l}^{ \pm}, E_{y, r}^{ \pm}$by formula (6.3).

14: end for

\section{end}

We note that, in Step 6, "left" and "right" can be distinguished by the $x_{1}$ coordinate, because of Definition (3.1). If $z_{y, l}^{-}$(as well $z_{y, r}^{-}$) and $y$ belong to $\Gamma$, then they constitute two vertexes of precisely one element in $\mathcal{M}^{-}$, the same for the superscript + . The computation of the gradient in Step 13 will be explained in detail for each experiment. 
Finally we remark that in our geometric configuration the observation $\Gamma_{o}$ and the Dirichlet part $\Gamma_{D}$ of the boundary are fixed. Therefore formula (5.10) reads

$$
\left.\frac{d}{d t} J\left(u_{t}, \Gamma_{t}\right) \theta\right|_{t=0}=\left.\llbracket g v \rrbracket \theta_{\mathrm{t}}\right|_{\bar{\Gamma} \cap \Gamma_{N}}+\int_{\Gamma} \llbracket \nabla u \cdot \nabla v \rrbracket(\theta \cdot n) d s .
$$

\section{Numerical experiments}

We carried out three numerical experiments to exploit the performance of our scheme in diverse situations, for different true breaking lines $\Gamma_{T}$, admissible velocities $\theta$ and traction forces $g$.

In all the three experiments the observation part of the boundary is fixed to $\Gamma_{o}=\Gamma_{N}^{+} \cup \Gamma_{N}^{-}$so that $\bar{\Gamma} \cap \Gamma_{o}=\emptyset$. We recall the notation $x^{0}, \ldots, x^{m}$ for the piece-wise linear discretization $\mathrm{M}$ of $\Gamma$, where the velocity is computed.

\subsection{Example 1}

As a first experiment we consider the following particular case of traction force $g$ such that the third condition in (3.2) explicitly reads

$$
\nabla u \cdot n=-1 \text { on } \Gamma_{N}^{-}, \quad \nabla u \cdot n=1 \text { on } \Gamma_{N}^{+}, \quad \nabla u \cdot n=0 \text { on } \Gamma_{N}^{0} .
$$

We set the corresponding velocity field as

$$
\theta \cdot n=-\llbracket \nabla u \cdot \nabla v \rrbracket, \quad \theta_{\mathrm{t}}=0 \text { on } \Gamma .
$$

Then (6.4) turns into

$$
\left.\frac{d}{d t} J\left(u_{t}, \Gamma_{t}\right) \theta\right|_{t=0}=-\int_{\Gamma} \llbracket \nabla u \cdot \nabla v \rrbracket^{2} d s<0 .
$$

The velocity in Step 13 is updated according to (7.2) by the following element wise formula

$$
\theta_{i}(\Gamma)=\left(\theta_{i}^{-}-\theta_{i}^{+}\right) \quad i=0, \ldots, m,
$$

where on each finite interval $\left(x^{i-1}, x^{i}\right), i=1, \ldots, m$ we set the piece-wise constant mean

$$
\theta_{i-1}^{ \pm}=\theta_{i}^{ \pm}=\frac{1}{2}\left[\left.(\nabla u \cdot \nabla v)\right|_{E_{x^{i-1, r}}^{ \pm}}+\left.(\nabla u \cdot \nabla v)\right|_{E_{x^{i}, l}^{ \pm}}\right],
$$

and the gradients of $u$ and $v$ are computed by Algorithm 2. The approximation (7.5) of (7.2) is exact when the meshes $\mathcal{M}^{ \pm}$coincide with $M$ on $\Gamma^{ \pm}$. Then we update the breaking line by a continuous piece-wise linear curve obtained from the 
mean value at $x^{i}, i=1, \ldots, m-1$, where differences between $\theta_{i}^{ \pm}$on $\left(x^{i-1}, x^{i}\right)$ and $\theta_{i}^{ \pm}$on $\left(x^{i}, x^{i-1}\right)$ happen.

First we remark that by the third equality of (7.1) we have

$$
0=g=\nabla u \cdot n=\nabla u \cdot(1,0)=\frac{\partial u}{\partial x_{1}} \text { on } \Gamma_{N}^{0}
$$

and by the fourth of (3.2) we have

$$
0=\nabla u \cdot n^{ \pm}=\nabla u \cdot(0, \pm 1)=\frac{\partial u}{\partial x_{2}} \text { on } \Gamma^{ \pm} .
$$

By the previous statement and the first of (7.2) we get the velocity at the right tip

$$
\theta \cdot n=0 \text { on } \bar{\Gamma} \cap \Gamma_{N} \text {. }
$$

Therefore, in this configuration, the tip of a breaking line initialization $\Gamma^{0}$ cannot be moved by iterations of $\theta$ along the Neumann boundary.

The first experiment was carried out for the piece-wise linear $\Gamma_{T}$ shown in Fig. 1 (a), defined as $\Gamma_{T}=[0,0.25 ; 0.5,0.25 ; 1.5,0 ; 2,0]$. The experiment was carried out with $m=2^{5}$ points in the discretization of $\mathrm{M}$, and time step $\tau=2 h$. In Fig 1 (b) the true solution is shown, i.e. $z$ the solution to (6.2) for $\Gamma_{T}$ as in Fig. 1 (a). In Fig. 2 the current breaking line $\Gamma^{i}$ is represented corresponding to the initialization $\Gamma^{0}$ and selected iterations $i=0,4,10,15$. The corresponding solution $u^{i}$ and the adjoint $v^{i}$ recovered by Algorithm 1 are shown in Fig. 3 and Fig. 4, respectively. In Fig. 1 (b) we note that the solution $z$ is symmetric with respect to the initial breaking line $\Gamma^{0}$; on the contrary the adjoint $v^{i}$ shows an asymmetric behaviour, due to the influence of the observation datum $z$ in the Neumann boundary condition on $\Gamma_{o}$ (see (4.2)). In Fig. 1, Fig. 3, and Fig. 4 we see also that the Dirichlet and Neumann boundary conditions (plotted in cyan and yellow) are attained, as expected.

In Fig. 5, Fig. 6, Fig. 7, and Fig. 8, we show the first and second component of the gradient of $u^{i}$ ( $v^{i}$, respectively). In Fig. 5 and Fig. 7 we note again that the Neumann boundary conditions (plotted in cyan and yellow) are attained on $\Gamma_{o}$, as expected. Moreover, the first component of the gradient of $u^{i}$ and $v^{i}$ is zero, according to (7.6).

In Fig. 9 we show the normalized graphic of $\theta$ on the current breaking line represented in Fig. 2. The violet and blue coloured elements are the elements where the velocity is computed in the upper and lower domains, according to (7.5). Note that in $\overline{\Gamma^{i}} \cap \Gamma_{N}$ the velocity is always zero, according to (7.7).

The iterations results are gathered in Fig. 10. From Fig. 10 (a) we can see that the true breaking line is recovered quite well. Moreover in plot 10 (b) we show 
the $\ell^{2}$-distance Err between the recovered breaking line and the true one, and, in plot (c), the values of the objective functional $J$ in (3.3) along the iterates. While Err decreases with small oscillations (local minima), $J$ decreases monotonically to zero, as expected, and their values reached after $K_{\max }=20$ iterations are $\mathrm{Err}_{\min }=0.02$, and $\mathrm{J}_{\min }=0.01$, respectively.

We mention that the same kind of results were obtained for other $\Gamma_{T}$ tested, and the right tip $\overline{\Gamma^{i}} \cap \Gamma_{N}$ has not moved during the iterations. Otherwise, if $\overline{\Gamma^{i}} \cap \Gamma_{N} \neq$ $(2,0)$, then the velocity in (7.2) cannot follow it, as expressed in (7.7). A remedy of this situation is to account for $g \neq 0$ at $\overline{\Gamma^{i}} \cap \Gamma_{N}$, according to (7.6).

In the following experiments, we focus on the case in which the right tip on the Neumann boundary will be moved during the iterations. Since by (7.7) we cannot expect to be able to recover a breaking line with a moving right tip, as explained before, we change the traction force $g$.

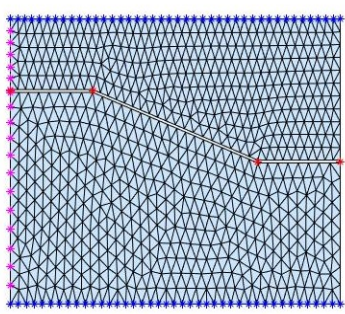

(a)

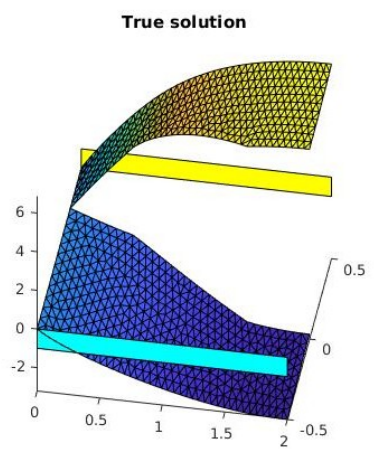

(b)

Figure 1. First experiment. (a): true breaking line $\Gamma_{T}$; (b): true solution $z$.
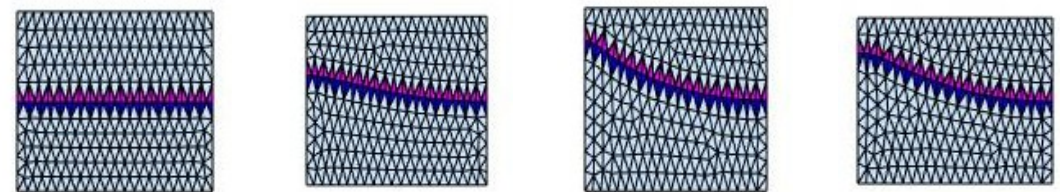

Figure 2. First experiment. Elements in the current fracture $\Gamma^{i}$ where the velocity is computed, for $i=0,4,10,15$ (from left to right). 


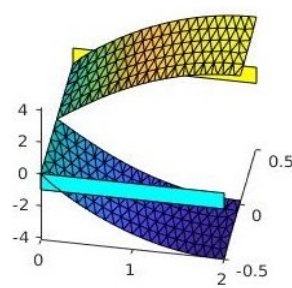

(a)

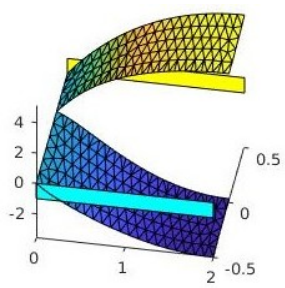

(b)

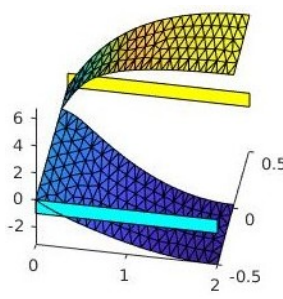

(c)

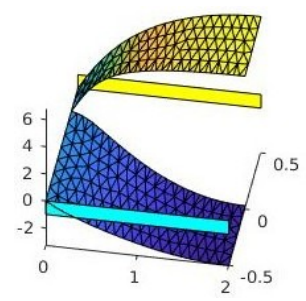

(d)

Figure 3. First experiment. Solution $u^{i}, i=0,4,10,15$ (from left to right).

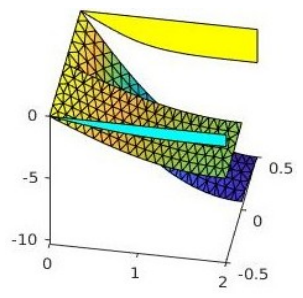

(a)

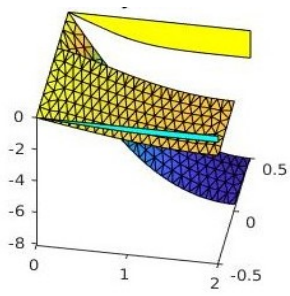

(b)

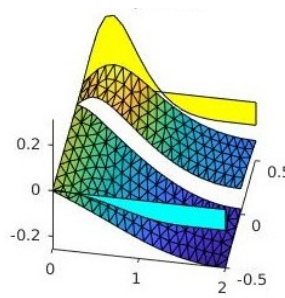

(c)

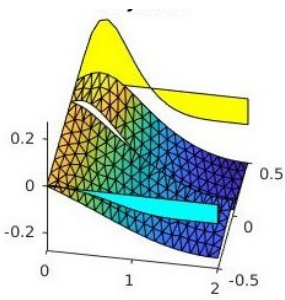

(d)

Figure 4. First experiment. Adjoint $v^{i}, i=0,4,10,15$ (from left to right).
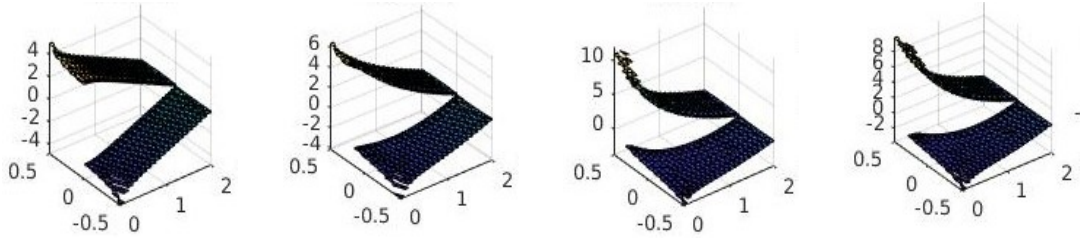

Figure 5. First experiment. Gradient of $u^{i}$, first component, in: $\Omega^{0}, \Omega^{4}, \Omega^{10}, \Omega^{15}$ (from left to right).

\subsection{Example 2}

As a second experiment we consider the line segment $\Gamma_{T}=[0,0.5 ; 2,-0.5]$ and $M$ consisting of two end points (that is, $m=1$ ) and the traction force $g$ so that

$$
\nabla u \cdot n=0 \text { on } \Gamma_{N}^{-}, \quad \nabla u \cdot n=0 \text { on } \Gamma_{N}^{+}, \quad \nabla u \cdot n=1 \text { on } \Gamma_{N}^{0} .
$$



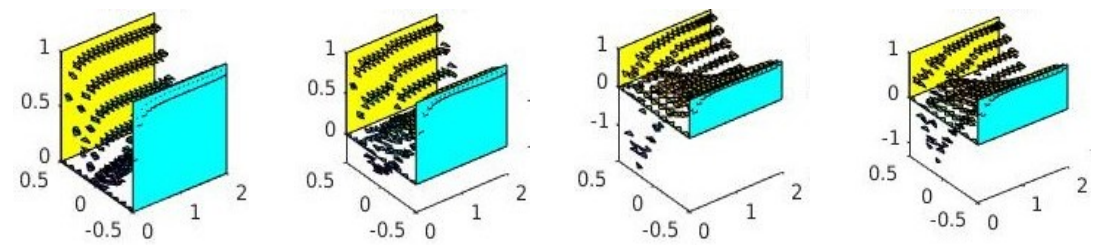

Figure 6. First experiment. Gradient of $u^{i}$, second component, in: $\Omega^{0}, \Omega^{4}, \Omega^{10}, \Omega^{15}$ (from left to right).
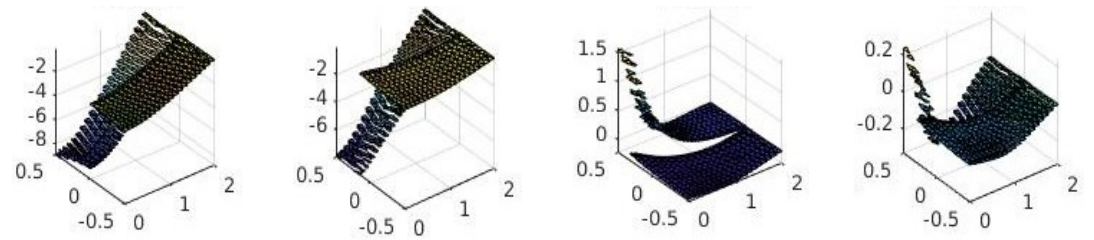

Figure 7. First experiment. Gradient of $v^{i}$, first component, in: $\Omega^{0}, \Omega^{4}, \Omega^{10}, \Omega^{15}$ (from left to right).
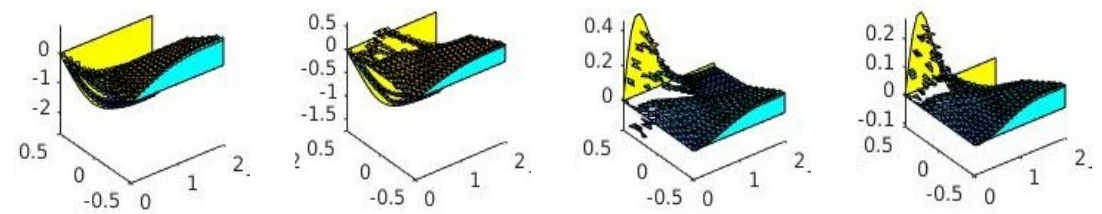

Figure 8. First experiment. Gradient of $v^{i}$, second component, in: $\Omega^{0}, \Omega^{4}, \Omega^{10}, \Omega^{15}$ (from left to right).
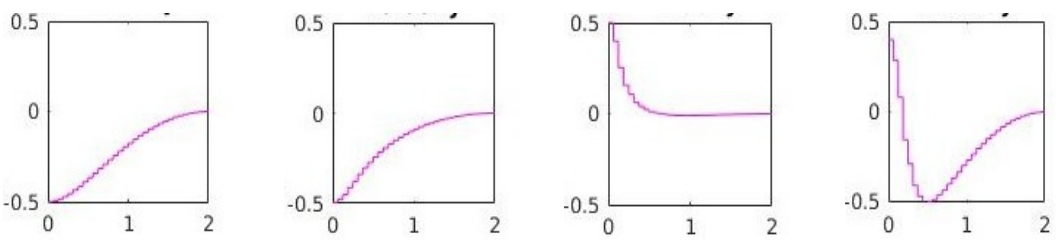

Figure 9. First experiment. Velocity $\theta^{i}$, for $i=0,4,10,15$ (from left to right). 


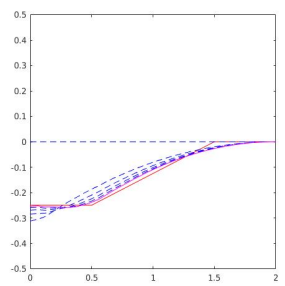

(a)

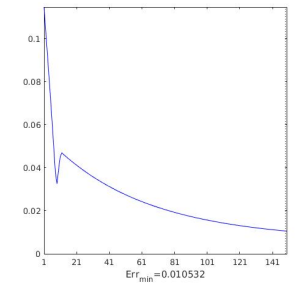

(b)

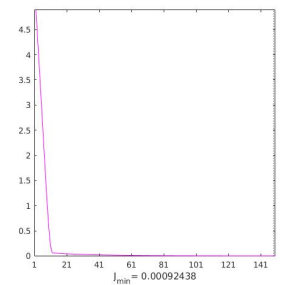

(c)

Figure 10. First experiment. From left to right: (a) true breaking line (red line), approximated breaking line (blue dotted line), final recovered breaking line (violet line). (b) $\ell^{2}$-distance error between the true breaking line and the recovered one. Err $_{\min }$ is its minimal value. (c) Value of the functional $J$ in (3.3) along the iterates. $J_{\min }$ is its minimal value.

Since $g$ does not vanish on $\Gamma_{N}^{0}$, the first term in (6.4) should be negative. Therefore, the velocity in (7.2) is refined according to

$$
\theta \cdot n=-\llbracket \nabla u \cdot \nabla v \rrbracket, \quad \theta_{\mathrm{t}}=0 \text { on } \Gamma, \quad \theta_{\mathrm{t}}=-w \llbracket g v \rrbracket \text { on } \bar{\Gamma} \cap \Gamma_{N},
$$

where the influence of the value $\theta_{\mathrm{t}}$ at the right tip was weighted by a factor $w>0$. The purpose of this experiment was to determine numerically the weight $w$ at $\bar{\Gamma} \cap \Gamma_{N}$.

Then, the velocity in Step 13 of Algorithm 2 on intervals $\left(x^{i-1}, x^{i}\right), i=1, \ldots, m$ is updated by (7.8), where now $\theta$ is defined element-wise as

$$
\begin{aligned}
& \theta_{i}(\Gamma)=\theta_{i}^{-}-\theta_{i}^{+} \text {for } i=0, \ldots, m-1, \\
& \qquad \theta_{m}(\Gamma)=\frac{1}{2}\left[\theta_{m}^{-}-\theta_{m}^{+}+w\left(v^{-}\left(x^{m}\right)-v^{+}\left(x^{m}\right)\right)\right],
\end{aligned}
$$

where $\theta_{i}^{ \pm}$are defined in (7.5).

Diverse values of $w$ were tested. In Fig. 11 we report the best result that we got in the case of $w=1 / \sqrt{h}$. As we can see from Fig. 11 (a), the breaking line is recovered almost perfectly with an $\operatorname{Err}_{\min }$ of the order of $10^{-4}$ after $K_{\max }=50$ iterations with time step $\tau=7 h$.

Note that differently from the first experiment, both the left and right tips $\Gamma_{T}$ are moved during the iteration. However, the geometry of $\Gamma_{T}$ shown in Fig. 11 is straight, defined by only two points. This is mainly due to the observed fact that the local basis in which the velocity is searched did not perform well to recover shape, since the right tip was moving along $\Gamma_{N}$. To improve this situation we carried out a third experiment where we used a global basis for the velocity as described in the next subsection. 


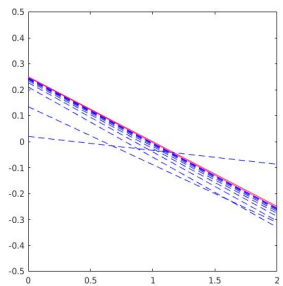

(a)

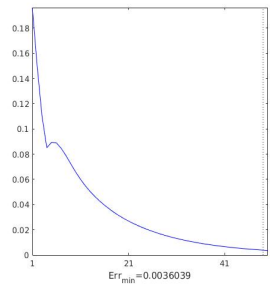

(b)

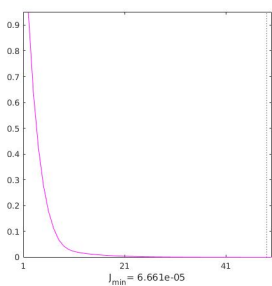

(c)

Figure 11. Second experiment. From left to right: (a) true breaking line (red line), approximated breaking line (blue dotted line), final recovered breaking line (violet line). (b) $\ell^{2}$-distance error between the true breaking line and the recovered one. Err $_{\min }$ is its minimal value. (c) Value of the functional $J$ in (3.3) along the iterates. $J_{\min }$ is its minimal value.

\subsection{Example 3}

In the third experiment, differently from the first and second one, we use a global trigonometric basis and choose for the normal velocity at $\Gamma$ the ansatz

$$
\theta \cdot n=\sum_{k=0}^{2 m} c_{k} L_{k}(x)
$$

with unknown coefficients $c_{k}, k=0, \ldots, 2 m$, and the trigonometric basis

$$
L_{k}(x)=\cos (k x), k=0, \ldots, m, L_{k}(x)=\sin (k x), k=m+1, \ldots, 2 m .
$$

We insert the ansatz in the shape derivative and consider

$$
\begin{aligned}
\left.\frac{d}{d t} J\left(u_{t}, \Gamma_{t}\right) \theta\right|_{t=0} & =\int_{\Gamma}(\theta \cdot n) \llbracket \nabla u \cdot \nabla v \rrbracket d s \\
& =\sum_{k=0}^{2 m} c_{k}\left\{\left.\sum_{i=1}^{m} \llbracket \nabla u \cdot \nabla v \rrbracket\right|_{\left(x^{i-1}, x^{i}\right)} I_{k, i}\right\},
\end{aligned}
$$

where we denote for $k=0, \ldots, 2 m$ and $i=1, \ldots, m$ the entries

$$
I_{k, i}:=\int_{x^{i-1}}^{x^{i}} L_{k}(x) d s .
$$

We set for $k=0, \ldots, 2 m$

$$
c_{k}=-\left.\sum_{i=1}^{m} \llbracket \nabla u \cdot \nabla v \rrbracket\right|_{\left(x^{i-1}, x^{i}\right)} I_{k, i},
$$


and the mean value interpolation according to (7.5) and (7.8):

$$
\begin{aligned}
& \left.\llbracket \nabla u \cdot \nabla v \rrbracket\right|_{\left(x^{i-1}, x^{i}\right)}=\left.w \llbracket g v \rrbracket\right|_{\bar{\Gamma} \cap \Gamma_{N}} \delta_{\left[x^{i-1}, x^{i}\right] \cap \Gamma_{N}} \\
+ & \frac{1}{2}\left[\left.(\nabla u \cdot \nabla v)\right|_{E_{x^{i-1}, r}^{+}}-\left.(\nabla u \cdot \nabla v)\right|_{E_{x^{i-1}, r}^{-}}+\left.(\nabla u \cdot \nabla v)\right|_{E_{x^{i}, l}^{+}}-\left.(\nabla u \cdot \nabla v)\right|_{E_{x^{i}, l}^{-}}\right],
\end{aligned}
$$

with the indicator function $\delta_{\left[x^{i-1}, x^{i}\right] \cap \Gamma_{N}}=1$ for $i=m$ and zero otherwise. From (7.11) and (7.12) we get a descent direction since

$$
\left.\frac{d}{d t} J\left(u_{t}, \Gamma_{t}\right) \theta\right|_{t=0}=-\sum_{i=1}^{m} c_{i}^{2}<0 .
$$

In order to compute the gradients in (7.13), we use Algorithm 2.

The first test was carried out for the piece-wise linear $\Gamma_{T}$ showed in Fig. 12 (a), defined as $\Gamma_{T}=[0,0.25 ; 0.5,0.25 ; 1.5,-0.25 ; 2,-0.25]$, and the corresponding solution $z$ depicted in plot (b). We remark that in this case the cosine basis is sufficient for the geometry tested. In Algorithm 1 we update $\Gamma^{i+1}$ in Step 12 with

$$
\theta\left(\Gamma^{i}\right)=\sum_{k=0}^{m} c_{k} L_{k}(x) .
$$

In Fig. 13 and 14, we see the evolution of the breaking line and the velocity, respectively, at the selected steps $i=4,7,9,11$. Note that the left and right tips are almost perfectly identified within the first 7 iterations. Subsequently the velocity decreases almost to zero in the right tip, corresponding to very small changes in the breaking line identification. In Fig. 14 we see how the velocity changes its shape during the iterations. As in Fig. 2, each group of 4 coloured elements corresponds to one of the $m=4$ points in which the velocity is computed. The violet and yellow are the elements in the upper domain, the blue and green are the ones in the lower domain. In Fig. 15 we report the result we got for $m=4, \tau=4 h$. As we can see from Fig. 15 (a), both tips of the breaking line are perfectly recovered (as expected) in just 12 iterations. Moreover, the shape of the breaking line is better recovered, compared to the result we get with the same $\Gamma_{T}$, but with a local basis for the velocity (as used in the previous two experiments). The $\operatorname{Err}_{\min }$ is of the order of $10^{-2}$ and both the Err and the value of the functional $\mathrm{J}$ are decreasing with the iterations, see Figure 16.

As second test case, we consider $\Gamma_{T}=[0,0 ; 0.5,0 ; 1,0 ; 1.5,-0.25 ; 2-0.25]$, with moving right tip and fixed left one, where the left tip at the Dirichlet boundary is not moved during the iteration. In this case, to recover the breaking line, we used 


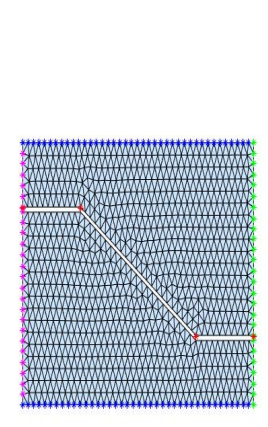

(a)

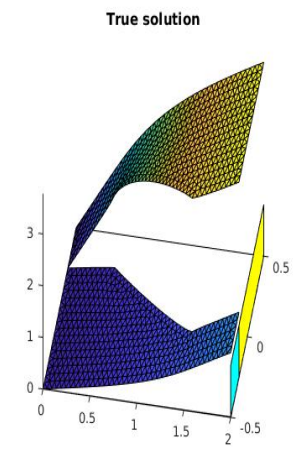

(b)

Figure 12. Third experiment. (a): true breaking line $\Gamma_{T}$; (b): true solution $z$.
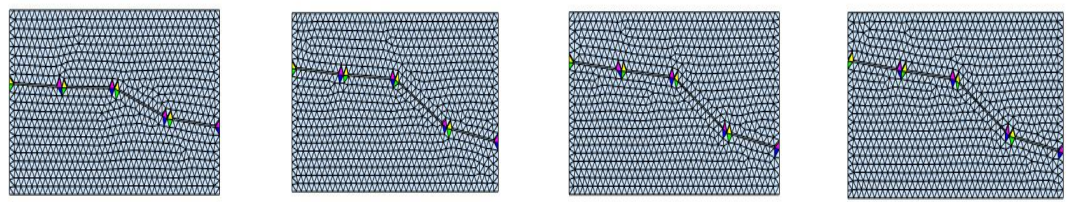

Figure 13. Third experiment. Elements in the current fracture $\Gamma^{i}$ where the velocity is computed, for $i=4,7,9,11$ (from left to right).
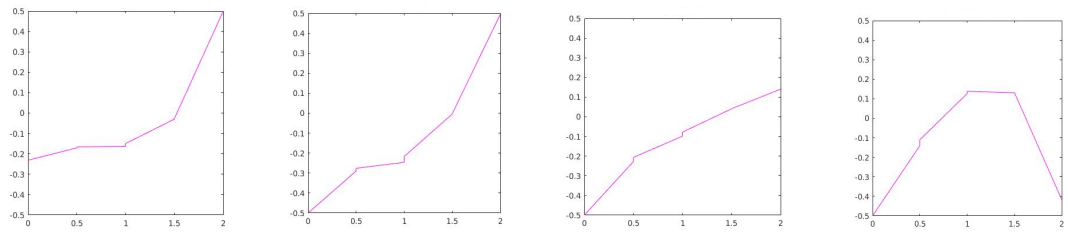

Figure 14. Third experiment. Velocity $\theta^{i}$, for $i=4,7,9,11$ (from left to right).

the sine and cosine trigonometric basis defined in (7.10). As we can see from Fig. 16 the breaking line is very well recovered within 150 iterations. 


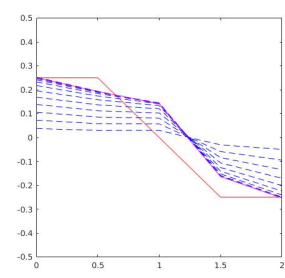

(a)

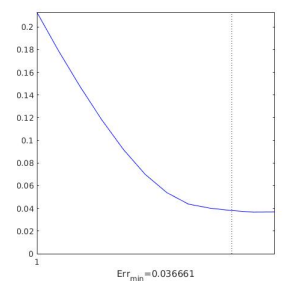

(b)

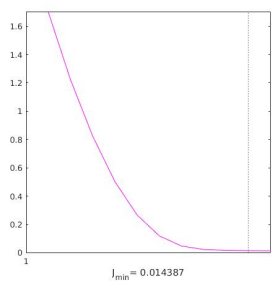

(c)

Figure 15. Third experiment, second test case. From left to right: (a) true breaking line (red line), approximated breaking line (blue dotted line), final recovered breaking line (violet line). (b) $\ell^{2}$-distance error between the true breaking line and the recovered one. Err $_{\min }$ is its minimal value. (c) Value of the functional $J$ in (3.3) along the iterates. $J_{\min }$ is its minimal value.

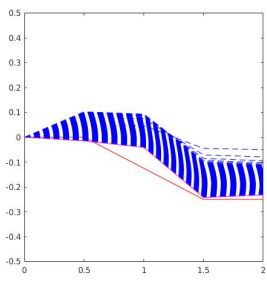

(a)

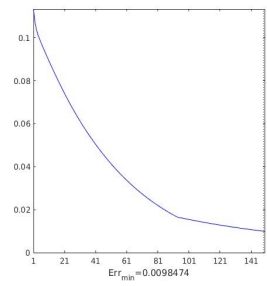

(b)

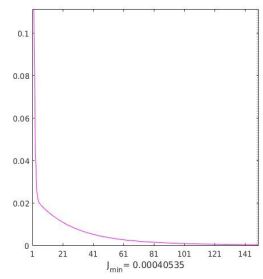

(c)

Figure 16. Third experiment. From left to right: (a) true breaking line (red line), approximated breaking line (blue dotted line), final recovered breaking line (violet line). (b) $\ell^{2}$-distance error between the true breaking line and the recovered one. Err $_{\text {min }}$ is its minimal value. (c) Value of the functional $J$ in (3.3) along the iterates. $J_{\min }$ is its minimal value.

Acknowledgments. V. A. Kovtunenko thanks Austrian Science Fund (FWF) project P26147-N26:PION, the RFBR and JSPS research project 16-51-50004 for partial support.

\section{Bibliography}

[1] C.C. Baniotopoulos, J. Haslinger and Z. Moravkova, Mathematical modeling of delamination and nonmonotone friction problems by hemivariational inequalities, Appl. Math. 50 (2005), 1-25. 
[2] M. Bonnet, A general boundary-only formula for crack shape sensitivity of integral functionals, C. R. Acad. Sci. Paris Ser. II b 327 (1999), 1215-1221.

[3] F. Cakoni and V.A. Kovtunenko, Topological optimality condition for the identification of the center of an inhomogeneity, Inverse Probl. 34 (2018), 035009.

[4] L. Chen, Programming of finite element methods in matlab, arXiv:1804.05156, 2018.

[5] M.C. Delfour and J.-P. Zolésio, Shape and Geometries: Metrics, Analysis, Differential Calculus, and Optimization, SIAM, Philadelphia, 2011.

[6] M. Fornasier, R. Ward, Iterative thresholing meets free-discontinuity problems, Found. Comput. Math., 10 (2015), 527-567.

[7] G. Fremiot, J. Sokolowski, Shape sensitivity analysis of variational problems in domains with cracks, Research Report, 3701, INRIA, 1999.

[8] B. Führ, V. Schulz and K. Welker, Shape optimization for interface identification with obstacle problems, Vietnam J. Math. 46 (2018), 967.

[9] D. Ghilli, K. Kunisch, On monotone and primal-dual active set schemes for $\ell^{p}$ - type problems, $p \in(0,1]$, Comput. Optim. Appl. , 72 (2019), 45-48.

[10] D. Ghilli, K. Kunisch, On a monotone scheme for nonconvex nonsmooth optimization with applications to fracture mechanics, JOTA, (2019), doi: 10.1007/s10957019-01545-4.

[11] M.S. Gockenbach, Understanding and Implementing the Finite Element Method, SIAM Philadelphia 2006.

[12] P. Grisvard, Singularities in Boundary Value Problems, Springer-Verlag, Berlin 1992.

[13] A. Hauptmann, M. Ikehata, H. Itou and S. Siltanen, Revealing cracks inside conductive bodies by electric surface measurements, Inverse Probl. 35 (2019), 025004.

[14] M. Hintermüller, V.A. Kovtunenko and K. Kunisch, Obstacle problems with cohesion: A hemi-variational inequality approach and its efficient numerical solution, SIAM J. Optim. 21 (2011), 491-516.

[15] K. Ito and K. Kunisch, Lagrange Multiplier Approach to Variational Problems and Applications, SIAM, Philadelphia, 2008.

[16] K. Ito, K. Kunisch and G. Peichl, Variational approach to shape derivatives, ESAIM: COCV 14 (2008), 517-539.

[17] S.I. Kabanikhin, Definitions and examples of inverse and ill-posed problems, J. Inv. Ill-Posed Problems, 16, (2008), 317-357.

[18] A.M. Khludnev and V.A. Kovtunenko, Analysis of Cracks in Solids, WIT-Press, Southampton, Boston, 2000. 
[19] A.M. Khludnev, A.A. Novotny, J. Sokolowski and A. Zochowski, Shape and topology sensitivity analysis for cracks in elastic bodies on boundaries of rigid inclusions, J. Mech. Phys. Solids 57 (2009), 1718-1732.

[20] V.A. Kovtunenko, A hemivariational inequality in crack problems, Optimization, 60, (2011), 1071-1089.

[21] V.A. Kovtunenko, Mathematical model of crack diagnosis: inverse acoustic scattering problem and its high-precision numerical solution, Vibroengineering PROCEDIA 22 (2019), 31-35.

[22] V.A. Kovtunenko and K. Kunisch, Problem of crack perturbation based on level sets and velocities, Z. angew. Math. Mech. 87 (2007), 809-830.

[23] V.A. Kovtunenko and K. Kunisch, High precision identification of an object: Optimality-conditions-based concept of imaging, SIAM J. Control Optim. 52 (2014), 773-796.

[24] V.A. Kovtunenko and K. Ohtsuka, Shape differentiability of Lagrangians and application to overdetermined problems, In: H. Itou, S. Hirano, M. Kimura, V.A. Kovtunenko and A.M. Khludnev (eds.) Mathematical Analysis of Continuum Mechanics and Industrial Applications III (Proc. CoMFoS18), Ser. Mathematics for Industry, Springer, Singapur, accepted.

[25] A. Laurain and K. Sturm, Distributed shape derivative via averaged adjoint method and applications, ESAIM: M2AN 50 (2016), 1241-1267.

[26] G. Leugering, M. Prechtel, P. Steinmann and M. Stingl, A cohesive crack propagation model: mathematical theory and numerical solution, Commun. Pure Appl. Anal. 12 (2013), 1705-1729.

[27] M. Nagumo, Über die Lage der Integralkurven gewöhnlicher Differentialgleichungen, Proc. Phys.-Math. Soc. Japan 3, (1942), 24:551-559.

[28] N. Nishimura, S. Kobayashi, Determination of cracks having arbitrary shapes with the boundary integral equation method, Eng. Anal. Bound. Elem., 15, (1995), 189195.

[29] N. Ovcharova and J. Gwinner, A Study of regularization techniques of nondifferentiable optimization in view of application to hemivariational inequalities, J. Optim. Theory Appl. 162 (2014), 754-778.

[30] E.M. Rudoy, First-order and second-order sensitivity analyses for a body with a thin rigid inclusion, Math. Meth. Appl. Sci. 39 (2016), 4994-5006.

[31] J. Sokolowski, J.-P. Zolesio, Introduction to Shape Optimization: Shape Sensitivity Analysis, Springer Series in Computational Mathematics, Springer-Verlag, 1991.

Received ???. 


\section{Author information}

Daria Ghilli, Institute for Mathematics and Scientific Computing, University of Graz, NAWI Graz, Heinrichstrasse 36, 8010 Graz, Austria.

E-mail: daria.ghilli@uni-graz.at

Karl Kunisch, Institute for Mathematics and Scientific Computing, University of Graz, NAWI Graz, Heinrichstrasse 36, 8010 Graz.

RICAM Institute, Altenbergerstrassee 69, 4040 Linz, Austria.

E-mail: karl.kunisch@uni-graz .at

Victor A. Kovtunenko, Institute for Mathematics and Scientific Computing, University of Graz, NAWI Graz, Heinrichstrasse 36, 8010 Graz.

Lavrentyev Institute of Hydrodynamics, Siberian Branch of the Russian Academy of Sciences, 630090, Novosibirsk, Russia.

E-mail: victor.kovtunenko@uni-graz.at 